\title{
Distribution of Residence Times of Time-Delayed Bistable Systems Driven by Noise
}

\author{
C. Masoller \\ Instituto de Física, Facultad de Ciencias, Universidad de la República, Igua 4225, Montevideo 11400, Uruguay
}

(Received 11 July 2002; published 17 January 2003)

\begin{abstract}
I study bistable time-delayed feedback systems driven by noise. Based on a two-state model with transition rates depending on the earlier state of the system I calculate analytically the residence-time distribution function. I show that the distribution function has a detailed structure, reflective of the effect of the feedback. By using an adequate indicator I give evidence of resonant behavior in dependence on the noise level. I also predict that this feedback-induced effect might be observed in two well-known optical bistable systems.
\end{abstract}

DOI: 10.1103/PhysRevLett.90.020601

The behavior of a bistable system under the influence of noise is a problem relevant in domains as different as quantum optics and neurophysiology. The phenomenon termed "stochastic resonance" (SR), which is the optimal enhancement of a periodic signal by a bistable system driven by a certain level of noise, has received considerable attention in the past decade (for review, see [1]). Different quantities have been introduced to quantify SR, among them, the response amplitude, the signal-to-noise ratio, and the residence-time distribution function. Another related phenomenon that has received considerable attention is termed "coherence resonance" (CR), which is the appearance of regular pulses in an excitable system driven by a certain level of noise [2]. Recently, it has been shown that SR and CR are closely related: SR was found in an excitable optical system [3], and CR was found in a chaotic bistable system [4].

A great deal of research has been devoted to the problem of calculating the statistical distribution of the times at which a bistable system switches between two states (also called residence-time distribution or first-passagetime distribution). The study of this problem in bistable systems that have a time-delayed feedback mechanism has not been treated so far, to the best of my knowledge, probably due to the difficulties that arise from the nonMarkovian character of the dynamics induced by the time delay. However, systems described by delay-differential equations arise in many fields of research. Examples can be found in the context of neurophysiology, where neurons interact through synaptic connections, and there is a delay in the connection due to the finite transmission time along the axon, and to the finite response time at the synapse [5]. In cavity nonlinear optics, many effects depend on the finite velocity of light and require a description incorporating the delay due to the round-trip time of the light in the cavity [6,7].

Recently Tsimring and Pikovsky [8] studied the timedelayed bistable system

$$
\dot{x}=x(t)-x^{3}(t)+\epsilon x(t-\tau)+\sqrt{2 D} \xi(t),
$$

where $\epsilon$ is the feedback strength, $\tau$ is the delay time, $\xi(t)$ is a Gaussian white noise, and $D$ is the noise strength.
PACS numbers: 05.40.Ca, 05.45.-a, 42.65.Pc, 42.65.Sf

Based on a two-state model with transition rates depending on the earlier state of the system, they calculated the correlation function, the power spectrum, and gave evidence of CR and SR behavior.

In this Letter I show that the interplay of noise and time-delayed feedback might lead to new intriguing effects, which are unveiled studying the statistical distribution of residence times. First, I use the framework of the model of Ref. [8] to calculate analytically (under certain approximations) the residence-time distribution function, $\rho(T)$. I show that $\rho(T)$ exhibits a feedbackinduced structure which is resonant with the noise level. Second, I show that the feedback-induced structure can be observed in two well-known optical systems, namely, in a model for vertical-cavity surface-emitting lasers and in the vector complex Ginzburg-Landau equation.

First I focus on Eq. (1), which for small $\epsilon$ has two stable fixed points, $x^{ \pm}= \pm \sqrt{1+\epsilon}$, separated by a saddle point, $x_{s}=0$. Noise drives the system from the basin of attraction of one fixed point to the other (termed "wells" from now on). The residence time, $T$, for a particle in one well is the time between two consecutive crossings of the well barrier. In the absence of delayed feedback the residence times are statistically distributed according to

$$
\rho(T)=r_{K} \exp \left(-r_{K} T\right),
$$

where $r_{K}=\left(1 / \sqrt{2 \pi^{2}}\right) \exp [-(1 / 4 D)]$ is the Kramers's escape rate $[9,10]$. Figure 1 shows the distribution of residence times, calculated numerically by simulation of Eq. (1) with $\tau=15, \epsilon=0.05$ [Fig. 1(a)], and $\epsilon=-0.05$ [Fig. 1(b)]. We see that $\rho(T)$ decays exponentially, exhibiting a sharp discontinuity for residence times $T \sim \tau$. This behavior has to be contrasted with the monotonous exponential decay for $\epsilon=0$ given by Eq. (2).

To understand the structure of $\rho(T)$, which is reflective of the effect of the time-delayed feedback, I calculate $\rho(T)$ using the framework of the two-state model of Ref. [8], in which intrawell fluctuations are neglected and the dynamical variable $s(t)$ takes two values $s=$ \pm 1 corresponding to $x>0$ and $x<0$, respectively. The time-delayed feedback is introduced by considering two 

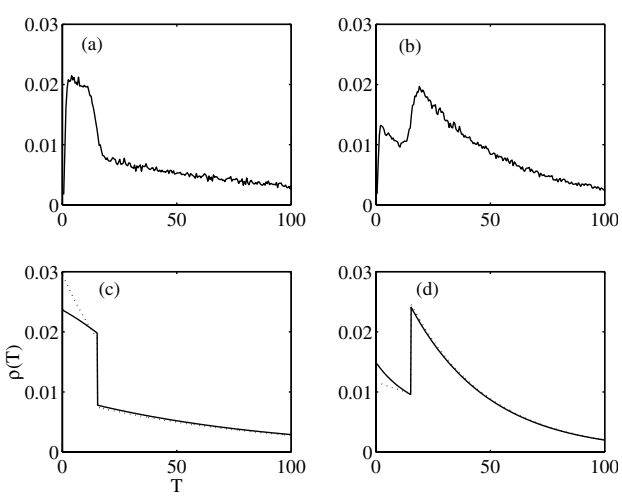

FIG. 1. (a),(b) Distribution of residence times calculated numerically integrating Eq. (1) for $D=0.1, \tau=15$, (a) $\epsilon=0.05$, (b) $\epsilon=-0.05$. (c),(d) Distribution of residence times calculated analytically using Eq. (10) (dashed line) and Eq. (12) (solid line). The parameters are the same as in (a),(b).

different switching rates, depending on the state $s(t-\tau)$ : $p_{1}$ if the past state at time $t-\tau$ is the same as at time $t$, and $p_{2}$ otherwise. For small $\epsilon$ and $D, p_{1}$ and $p_{2}$ can be calculated by virtue of the Kramers formula $r_{K}=(2 \pi)^{-1} \sqrt{U^{\prime \prime}\left(x^{ \pm}\right) U^{\prime \prime}\left(x_{s}\right)} \exp (-\Delta U / D) \quad$ with $U\left(x, x_{\tau}\right)=x^{4} / 4-x^{2} / 2-\epsilon x_{\tau}$, where the notation $x_{\tau}$ signifies that $x$ is evaluated at time $t-\tau$. The switching rates are given by [8]

$$
p_{1,2}=\frac{\sqrt{2 \pm 3 \epsilon}}{2 \pi} \exp \left(-\frac{1 \pm 4 \epsilon}{4 D}\right) .
$$

I follow earlier theories for calculating the residencetime distribution function in the case of stochastic resonance [11-14]: first I calculate the escape-time distribution function, $\rho^{ \pm}\left(T \mid t_{0}\right)$, which is the distribution of times the particle spends in one well, $T$, conditioned by the time it enters the well, $t_{0}$. In the following I consider, without loss of generality, the escape out of the right well, assuming that particles are absorbed after they escape; i.e., recrossing events are neglected. Assuming that a particle is in the left well for $t<t_{0}$ with $t_{0} \geq \tau$ and enters the right well at time $t_{0}$, the rate equation for the population of the right well, $p^{+}(t)$, is

$$
\begin{array}{ll}
\dot{p}^{+}(t)=-p_{2} p^{+}(t) & \text { if } t_{0} \leq t<t_{0}+\tau, \\
\dot{p}^{+}(t)=-p_{1} p^{+}(t) & \text { if } t \geq t_{0}+\tau .
\end{array}
$$

Here I have used the fact that in the interval $\left(t_{0}-\tau, t_{0}\right)$ no jumps occurred since the particle was in the left well. The above equations can be integrated with the initial condition $p^{+}\left(t_{0}\right)=1$ to give

$$
\begin{aligned}
& p^{+}(t)=\exp \left[-p_{2}\left(t-t_{0}\right)\right] \quad \text { if } t_{0} \leq t<t_{0}+\tau \\
& p^{+}(t)=\exp \left(-p_{2} \tau\right) \exp \left[-p_{1}\left(t-\tau-t_{0}\right)\right] \quad \text { if } t \geq t_{0}+\tau .
\end{aligned}
$$

Starting to measure the time when the particles enter the right well, the escape-time distribution function of 020601-2 the right well can be calculated as [12]

$$
\rho^{+}\left(T \mid t_{0}\right)=-\frac{1}{Z} \frac{d p^{+}(t)}{d t},
$$

where $Z$ is a normalization constant such that $\int_{0}^{\infty} \rho^{+}\left(T \mid t_{0}\right) d T=1$. From Eqs. (5) and (6)

$\rho^{+}\left(T \mid t_{0}\right)=p_{2} \exp \left(-p_{2} T\right) \quad$ if $T<\tau$,

$\rho^{+}\left(T \mid t_{0}\right)=p_{1} \exp \left[-\left(p_{2}-p_{1}\right) \tau\right] \exp \left(-p_{1} T\right) \quad$ if $T \geq \tau$.

The residence-time distribution function can be obtained by averaging the escape-time distribution by the probability that the system spends a time $t_{0}$ in the left well before entering the right well:

$$
\rho^{+}(T)=\frac{1}{Z} \int_{0}^{\infty} \rho^{+}\left(T \mid t_{0}\right) \rho^{-}\left(t_{0}\right) d t_{0},
$$

where $Z$ is a normalization constant such that $\int_{0}^{\infty} \rho^{+}(T) d T=1$. Since the two wells are symmetric, we can drop the "+" and "-" supraindices and write

$$
\rho(T)=\frac{1}{Z} \int_{0}^{\infty} \rho\left(T \mid t_{0}\right) \rho\left(t_{0}\right) d t_{0} .
$$

As a first approximation, let us assume that the particle spends long times in a single well and only occasionally jumps from one well to the other. In this case I neglect the fact that $\rho\left(T \mid t_{0}\right)$ was calculated with $t_{0} \geq \tau$ and assume it is valid for all $t_{0}$. Since $\rho\left(T \mid t_{0}\right)$ is actually independent of $t_{0}$, Eq. (9) gives

$$
\rho(T)=\rho\left(T \mid t_{0}\right)
$$

The dashed lines in Figs. 1(c) and 1(d) show $\rho(T)$ calculated according to Eq. (10), for the same parameters as in Figs. 1(a) and 1(b). In spite of the strong simplifying assumptions made, there is a remarkable agreement with the main features exhibited by the residence-time distribution function calculated numerically: $\rho(T)$ decays exponentially with $T$ and presents a sharp discontinuity at $T=\tau$.

To assess the validity of the approximations done, I calculate $\rho\left(T \mid t_{0}\right)$ with $t_{0}<\tau$ in the following way: I assume that in the interval $0 \leq t \leq \tau$ only one jump occurs. The particle is in the right well for $0 \leq t<\tau-t_{0}$, jumps to the left well at time $t=\tau-t_{0}$, and jumps back to the right well at time $t=\tau$. In this case, the rate equation for the population of the right well is

$$
\begin{array}{ll}
\dot{p}^{+}(t)=-p_{1} p^{+}(t) & \text { if } \tau \leq t<2 \tau-t_{0}, \\
\dot{p}^{+}(t)=-p_{2} p^{+}(t) & \text { if } 2 \tau-t_{0} \leq t<2 \tau, \\
\dot{p}^{+}(t)=-p_{1} p^{+}(t) & \text { if } t \geq 2 \tau .
\end{array}
$$

Using Eq. (6) I can calculate $\rho\left(T \mid t_{0}\right)$ with $t_{0}<\tau$, and $\rho(T)$ can be obtained from

020601-2 


$$
\begin{aligned}
\rho(T)= & \int_{0}^{\tau} \rho\left(T \mid t_{0}<\tau\right) \rho\left(t_{0}\right) d t_{0} \\
& +\int_{\tau}^{\infty} \rho\left(T \mid t_{0} \geq \tau\right) \rho\left(t_{0}\right) d t_{0}
\end{aligned}
$$

using the ansatz $\rho(T)=C_{1} \exp \left(-p_{1} T\right)$ if $T<\tau, \rho(T)=$ $C_{2} \exp \left(-p_{1} T\right)$ if $T \geq \tau$ and the normalization condition $\int_{0}^{\infty} \rho(T) d T=1$. The solid lines in Figs. 1(c) and 1(d) show the results: for the above parameters there is only a small modification for $T<\tau$. Notice that in the framework of the two-state model and the approximations done, the effect of the feedback is twofold: it increases (or decreases) the probability of short residence times, and it modifies the rate of exponential decay, $\rho(T) \sim \exp \left(-p_{1} T\right)$, for large $T$.

Figure 2 displays the effect of noise. As $D$ increases noise washes out the effect of the feedback. The effect of the time-delayed feedback can be quantified in terms of the quantity $\sigma$, which is the value of the discontinuity of $\rho(T)$ for $T=\tau$, normalized to the value of $\rho(\tau)$ in the absence of feedback, $r_{K} \exp \left(-r_{K} \tau\right) / Z$. Figure 3 shows that $\sigma$ goes through a maximum when the noise strength $D$ varies. There is no resonance with the feedback, since when $|\epsilon|$ increases $\sigma$ monotonously increases.

Next I show that the feedback-induced structure of $\rho(T)$ can be observed in a model for vertical-cavity surface-emitting lasers (VCSELs). Unlike edge-emitting lasers, VCSELs present a complex polarization behavior due to their cylindrical symmetrical structure. The output of a VCSEL is usually linearly polarized along one of two orthogonal directions associated with crystalline or stress orientations. When the VCSEL begins to lase one linear polarization dominates, and when the injection current is increased, in many devices the emission switches to the other polarization state (current-driven polarization switching). A different phenomenon is the polarization switching triggered by spontaneous emission noise (sto-
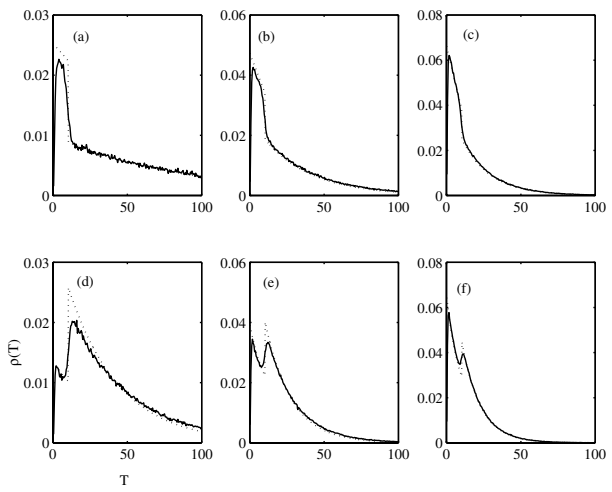

FIG. 2. Distribution of residence times for different feedback and noise. Solid line: $\rho(T)$ calculated numerically; dashed line: $\rho(T)$ calculated analytically using Eq. (12). The parameters are $\tau=10$ (a) $\epsilon=0.05, D=0.1$; (b) $\epsilon=0.05, D=0.15$; (c) $\epsilon=$ $0.05, D=0.2$; (d) $\epsilon=-0.05, D=0.1$; (e) $\epsilon=-0.05, D=$ 0.15 ; (f) $\epsilon=-0.05, D=0.2$. chastic polarization switching). In spite of the fact that the polarization of the light of a VCSEL is linked, not only to cavity anisotropies, but also to the field-material physics (to the angular momentum of the quantum states involved in the transitions for emission and absorption), it has been shown that stochastic polarization switching agrees well with the Kramers hopping problem [15,16]. Moreover, SR was observed in the polarized emission of a VCSEL, when a sinusoidal signal and noise were superimposed onto the injection current [17].

To investigate the effect of optical feedback I did simulations based on the San Miguel, Feng, and Moloney (SFM) model [18]:

$$
\begin{gathered}
\dot{E}_{ \pm}=\kappa(1+i \alpha)(N \pm n-1) E_{ \pm}-\left(\gamma_{a}+i \gamma_{p}\right) E_{\mp} \\
+\epsilon E_{ \pm}(t-\tau) \exp \left(-i \omega_{0} \tau\right)+\sqrt{\beta_{\mathrm{sp}}} \xi_{ \pm}, \\
\dot{N}=-\gamma_{N}\left[N-\mu+N\left(\left|E_{+}\right|^{2}+\left|E_{-}\right|^{2}\right)\right. \\
\left.+n\left(\left|E_{+}\right|^{2}-\left|E_{-}\right|^{2}\right)\right], \\
\dot{n}=-\gamma_{s} n-\gamma_{N}\left[N\left(\left|E_{+}\right|^{2}-\left|E_{-}\right|^{2}\right)+n\left(\left|E_{+}\right|^{2}+\left|E_{-}\right|^{2}\right)\right],
\end{gathered}
$$

where $E_{ \pm}$are the slowly varying complex amplitudes of the circularly polarized modes, $N$ is the total carrier difference between conduction and valence bands, and $n$ is the difference between the population inversions of the spin-up and spin-down channels. $\kappa$ is the field decay rate, $\gamma_{N}$ is the decay rate of $N$, and $\gamma_{s}$ is the spin-flip relaxation rate. $\gamma_{a}$ and $\gamma_{p}$ are the linear anisotropies (dichroism and birefringence, respectively). $\alpha$ is the linewidth enhancement factor and $\mu$ is the normalized injection current ( $\mu=1$ at threshold). $\tau$ is the external cavity round-trip time, $\epsilon$ is the feedback strength, and $\omega_{0} \tau$ is the feedback phase. $\beta_{\mathrm{sp}}$ is the spontaneous emission rate and $\xi_{ \pm}$are two uncorrelated complex Gaussian white noises.

I chose parameters such that the linearly polarized states, $x$ and $y$, are both stable. When a fluctuation large enough occurs, the laser switches from one state to the other (the intensities of the two modes are strongly anticorrelated, leaving the total intensity nearly constant during the switching). Because of the complex nature of the optical fields the effect of the feedback depends on the feedback phase, and due to the effect of anisotropies, the

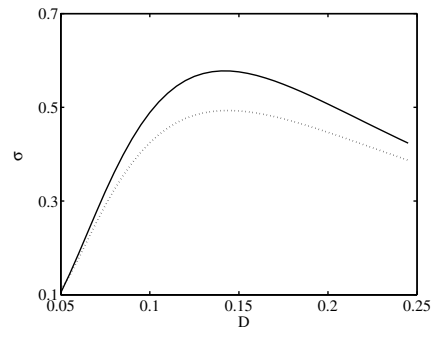

FIG. 3. $\sigma$ vs noise for $\tau=10, \epsilon=0.05$ (dashed line), $\epsilon=$ -0.05 (solid line). 

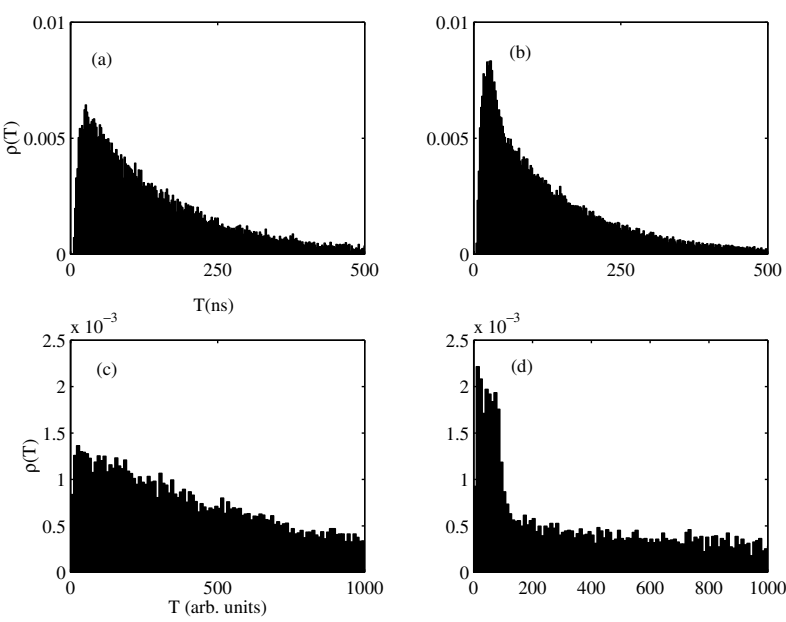

FIG. 4. (a),(b) Residence time distribution function for a VCSEL with weak optical feedback. The parameters are $\kappa=$ $300 \mathrm{~ns}^{-1}, \gamma_{N}=1 \mathrm{~ns}^{-1}, \gamma_{s}=50 \mathrm{~ns}^{-1}, \gamma_{a}=0, \gamma_{p}=3 \mathrm{~ns}^{-1}$, $\mu=1.1, \quad \alpha=3, \quad \tau=40 \mathrm{~ns}, \quad \omega_{0} \tau=0 \mathrm{rad}, \quad$ and $D=$ $3 \times 10^{-3} \mathrm{~ns}^{-1}$. (a) $\epsilon=0$; (b) $\epsilon=0.1 \mathrm{~ns}^{-1}$. (c),(d) Residence time distribution function for the vector complex GinzburgLandau equation with weak optical feedback. The parameters are $\beta=0.2, \gamma=2, \mu=0.3, \tau=100$, and $D=3 \times 10^{-3}$. (c) $\epsilon=0$; (d) $\epsilon=0.015$.

polarization modes have different mean residence times. Nevertheless, Figs. 4(a) and 4(b) show that the residencetime distribution function exhibits features similar to that of Eq. (1). With and without feedback $\rho(T)$ decays exponentially for large enough $T$, and with feedback short residence times are more probable.

Finally, to demonstrate that the above results are not restricted to the SFM model, I consider the vector complex Ginzburg-Landau equation, which gives a generic description of various types of lasers close to threshold [19]. Neglecting spatial terms and considering a weak feedback term the equation is

$$
\begin{aligned}
\dot{A}_{ \pm}= & \mu A_{ \pm}-(1+i \beta)\left(\left|A_{ \pm}\right|^{2}+\gamma\left|A_{\mp}\right|^{2}\right) A_{ \pm} \\
& +\epsilon A_{ \pm}(t-\tau)+\sqrt{\beta_{\mathrm{sp}}} \xi_{ \pm},
\end{aligned}
$$

where the parameter $\mu$ measures the distance to threshold, $\beta$ measures the detuning, and $\gamma$ measures the coupling between the right and left circularly polarized fields. $\epsilon, \tau, \beta_{\text {sp }}$, and $\xi_{ \pm}$have the same meaning as before. Solutions corresponding to circularly polarized light (i.e., $A_{ \pm}=Q_{ \pm} \exp \left[i\left(\omega_{ \pm} t+i \phi_{ \pm}\right)\right]$with either $Q_{+}=0$ or $\left.Q_{-}=0\right)$ are stable for $\gamma>1$. I focus on the region $\gamma>1$, where noise strong enough induces switching from one circularly polarized state to the other. Figures 4(c) and 4(d) display the distribution of residence times in one state, in the absence of feedback [Fig. 4(c)] and with weak feedback [Fig. 4(d)]. Again, the effect of optical feedback is to favor the short residence times.
To summarize, I have studied the distribution of residence times of bistable, noise-driven systems with time-delayed feedback. I demonstrated that with simple approximations the distribution of residence times can be analytically calculated. The theory predicts the key features found numerically: $\rho(T)$ decays exponentially with $T$ and presents a discontinuity for $T=\tau$. By using as an indicator the normalized value of the discontinuity, $\sigma$, I gave evidence of a resonance as $\sigma$ passes through a maximum at an optimal value of the noise. I have also shown that this feedback-induced effect can be observed in lasers with weak optical feedback.

In the presence of a time-delayed feedback mechanism noise might lead to unexpected novel effects, and I hope that the results reported in this Letter will stimulate the experimental investigation of the interplay of noise and feedback in nonlinear systems.

The author acknowledges stimulating discussions with C. Mirasso, J. Mulet, M. San Miguel, M. Sciamanna, and H. Wio. This work was supported in part by CSIC and PEDECIBA, Uruguay.

[1] L. Gammaitoni, P. Hänggi, P. Jung, and F. Marchesoni, Rev. Mod. Phys. 70, 223 (1998).

[2] A. S. Pikovsky and J. Kurths, Phys. Rev. Lett. 78, 775 (1997).

[3] F. Marino et al., Phys. Rev. Lett. 88, 040601 (2002).

[4] C. Palenzuela et al., Europhys. Lett. 56, 347 (2001).

[5] N. MacDonald, Biological Delay Systems: Linear Stability Theory (Cambridge University Press, Cambridge, England, 1989).

[6] P. Mandel, Theoretical Problems in Cavity Nonlinear Optics (Cambridge University Press, Cambridge, England, 1997).

[7] R. Lang and K. Kobayashi, IEEE J. Quantum Electron. 16, 347 (1980).

[8] L. S. Tsimring and A. Pikovsky, Phys. Rev. Lett. 87, 250602 (2001).

[9] H. A. Kramers, Physica (Utrecht) 7, 284 (1940).

[10] R. Roy, R. Short, J. Durnin, and L. Mandel, Phys. Rev. Lett. 45, 1486 (1980); J. Opt. Soc. Am. 70, 1622 (1980).

[11] B. S. McNamara and K. Wiesenfeld, Phys. Rev. A 39, 4854 (1989).

[12] T. Zhou et al., Phys. Rev. A 42, 3161 (1990).

[13] R. Löfstedt and S. N. Coppersmith, Phys. Rev. E 49, 4821 (1994).

[14] M. H. Choi et al., Phys. Rev. E 57, 6335 (1998).

[15] M. B. Willemsen et al., Phys. Rev. Lett. 82, 4815 (1999).

[16] M. Travagnin et al., Phys. Rev. A 56, 1497 (1997).

[17] G. Giacomelli, F. Marin, and I. Rabbiosi, Phys. Rev. Lett. 82, 675 (1999).

[18] M. San Miguel, Q. Feng, and J.V. Moloney, Phys. Rev. A 52, 1728 (1995).

[19] M. San Miguel, Phys. Rev. Lett. 75, 425 (1995). 